SUBJECT AREAS:

CHARACTERIZATION AND ANALYTICAL

TECHNIQUES

SCANNING ELECTRON MICROSCOPY

Received

30 January 2014

Accepted

27 June 2014

Published

16 July 2014

Correspondence and requests for materials should be addressed to

R.W.W. (Roger.

whatmore@tyndall.ie)

or N.P.

(Nikolay.petkov@

tyndall.ie)

\section{Absence: Statistical Analysis of Inclusions in Multiferroic Thin Films}

\author{
Michael Schmidt ${ }^{1,2}$, Andreas Amann ${ }^{1,3}$, Lynette Keeney ${ }^{1}$, Martyn E. Pemble ${ }^{1,2}$, Justin D. Holmes ${ }^{1,2,4}$, \\ Nikolay Petkov' ${ }^{1}$ \& Roger W. Whatmore ${ }^{1,2,5}$
}

${ }^{1}$ Tyndall National Institute, University College Cork, 'Lee Maltings', Dyke Parade, Cork, Ireland, ${ }^{2}$ Department of Chemistry, University College Cork, Cork, Ireland, ${ }^{3}$ School of Mathematical Sciences, University College Cork, Ireland, ${ }^{4}$ Centre for Research on Adaptive Nanostructures and Nanodevices, Trinity College Dublin, Dublin 2, Ireland, ${ }^{5}$ Department of Materials, Royal School of Mines, Imperial College London, South Kensington Campus, London SW7 2AZ, UK.

Assertions that a new material may offer particularly advantageous properties should always be subjected to careful critical evaluation, especially when those properties can be affected by the presence of inclusions at trace level. This is particularly important for claims relating to new multiferroic compounds, which can easily be confounded by unobserved second phase magnetic inclusions. We demonstrate an original methodology for the detection, localization and quantification of second phase inclusions in thin Aurivillius type films. Additionally, we develop a dedicated statistical model and demonstrate its application to the analysis of $\mathrm{Bi}_{6} \mathrm{Ti}_{2.8} \mathrm{Fe}_{1.52} \mathrm{Mn}_{0.68} \mathrm{O}_{18}$ (B6TFMO) thin films, that makes it possible to put a high, defined confidence level (e.g. 99.5\%) to the statement of 'new single phase multiferroic materials'. While our methodology has been specifically developed for magnetic inclusions, it can easily be adapted to any other material system that can be affected by low level inclusions.

\section{$1 / 1$} bsence of Evidence is not Evidence of Absence" is a truism which confounds many analytical assertions. The confident statement of certainty: "There was nothing there" leads to the obvious retort: "How do you know?". In many analyses, especially those using microscopes (optical or electron), it is only possible to look at restricted volumes, and the smaller the inclusion, or defect, or the lower the level of the impurity being sought, the smaller the volume that can reasonably be examined. The absence of any evidence for second phases, defects or impurities in these volumes can lead to the erroneous conclusion (perhaps driven by wishful thinking) that there are none in the sample as a whole, when in fact all that can be asserted with certainty was that there was nothing in the volumes examined at the limit of resolution for the technique used. And yet all is not lost, because if the detection thresholds of a particular method are well understood, the volumes examined are known and one makes some justifiable assumptions about the size distributions of second phase particles being sought, then one can apply statistical methods to determine the confidence level that surrounds such a statement. In other words, one can convert the assertion of "certainty-ofabsence" to one which gives a level of probability that the density of the sought-for impurity or second phase inclusion is below a certain critical level. Conversely, it is possible to apply such methods to the design of experiments which will yield any required level of confidence. For example, it is possible to say that if one requires a confidence of $99.5 \%$ that the density of a certain impurity or defect of known composition and/or size is below a certain critical level in a specimen, then given the sensitivities of the analytical methodologies being used, the statistical analysis can be used to determine how large a total volume needs to be examined.

If one considers different examples of materials analysis, there are different cases according to whether one is searching for impurity elements in a simple, single element material like silicon, trying to detect inclusions which are widely different in composition/structure from the parent matrix or looking for second phases that are similar in composition and/or structure to the parent. If the material is a pure element like silicon, for instance, claims with respect to a given level of purity can be verified relatively easily by using a host of different techniques such as SIMS (secondary ion mass spectrometry) and the level of impurities can be determined down to the level of $\mathrm{ppb}^{1-4}$. On the other hand, there are scanning methods which can locate inclusions and structural defects ${ }^{5,6}$, but, as commonly used, these are generally limited to detecting a minimum amount of $1-2$ weight $\%$ of a particular inclusion type $\mathrm{f}^{7}$. Atom probe tomography $(\mathrm{APT})^{8}$ and scanning transmission electron microscopy (STEM) 
coupled with energy dispersive X-ray spectroscopy (EDS/EDX) ${ }^{9}$ and/ or electron energy loss spectroscopy (EELS) ${ }^{10,11}$ provide techniques which can resolve single atoms spatially and elementally, but are limited to very small sample volumes (areas of a few $\mathrm{nm}^{2}$ within a single atomic layer such as graphene film for example). Hence they cannot deal with a statistical distribution of small numbers of such entities dispersed over tens of $\mu \mathrm{m}^{3}$. There is a significant probability that the examination of a small volume of material at the high detail needed to see such very small inclusions may easily miss them, simply because they are not present in the particular volume of material examined. In such a case it is prejudicial to say that such defects or inclusions are not present in the sample - one can only put a probability upon them for their density being below a certain critical level.

On the other hand, an aggressive search for new materials is in progress and the development of single-phase multiferroic (simultaneously ferromagnetic and ferroelectric) oxides is a good example of this. These materials are of considerable interest for future memory and sensing applications ${ }^{12-18}$. Here, the assertion that a new material is a single-phase, room-temperature multiferroic may be confounded by the presence of second-phase ferromagnetic inclusions that may have formed during the sample preparation. This is the single most critical factor in the analysis of such materials because the magnetic properties of the sample overall are highly susceptible to the presence of such inclusions. The difficulty in excluding second phase inclusions as being responsible for any observed ferromagnetism of a given sample is that some of the elements used to form the main phase could easily be responsible for formation of a ferromagnetic second phase. The differences between main and second phases could be in stoichiometry and/or crystal structure and both factors may have influence on the magnetic behaviour.

This report demonstrates an original methodology for the detection, localization and quantification of second phase inclusions in thin Aurivillius type films. Even when the compositional differences between inclusion and main phase are rather subtle, our methodology provides a viable approach to not only identifying the inclusions but also to their localization and further in-depth structural investigation.

Additionally, we develop a dedicated statistical model applied to the design of the analytical (EDX) measurements and demonstrate its application to the analysis of $\mathrm{Bi}_{6} \mathrm{Ti}_{2.8} \mathrm{Fe}_{1.52} \mathrm{Mn}_{0.68} \mathrm{O}_{18}$ (B6TFMO) thin films. We present a detailed account of the sample preparation, transmission electron microscopy (TEM) and energy dispersive $\mathrm{X}$-ray analysis (EDX) that was necessary to put a high, defined confidence level ( $>99.5 \%)$ to the statement of 'new single phase multiferroic material'.

While this methodology has been developed specifically to identify magnetic second phase inclusions, it can easily be applied to other material systems, given that the analytical measurement and statistical model is adapted to the special case, thus making the developed methodology comprehensively applicable. It closes an existing gap in the analysis of materials, between atomic scale analysis and the detection of obvious large scale (micron size) inclusions. Due to the fast pace of development in the hard- and software for elemental detection when using the electron beam as a probe, further development of the presented methodology can be sought by introducing necessary automation $^{19,20}$

\section{Statistical Analysis}

Our analysis requires a number of concepts from statistics, which we now introduce. We will start by analyzing the statistical significance of a single measurement of a given sample volume and at a fixed resolution, and then proceed to evaluate a series of measurements at varying volumes and resolutions. Finally we show how to conclude with a high, pre-set statistical confidence level (e.g. 99.5\%) that a physical effect (which in the case at hand is the magnetic remanence of the sample) is not caused by undetected inclusions or defects.
Single measurement. In general, it is impossible to prove with certainty that no inclusions exist in a given sample, unless the whole sample is scanned for inclusions. In order to nevertheless obtain statistically significant results, we use the method of statistical inference through the refutation of a null hypothesis. In the case at hand we assume that the level of concentration of a particular impurity exceeds a certain level, and then show that this assumption does not hold with a certain statistical level of confidence. More specifically, let us first assume that a particular type of second (or impurity) phase inclusion is present in the sample at a known (volume) density $\rho$ and let us further assume that the individual inclusions do not interact with each other and are therefore independently distributed. We measure the number of inclusions found in a scan of a small volume $V$, as shown schematically in figure 1 . The probability $P(N, \rho)$ of finding precisely $N$ inclusions is given by the Poisson distribution

$$
P(N, \rho)=\frac{(\rho V)^{N}}{N !} e^{-\rho V} .
$$

In particular, the probability that no inclusions are present in a scanned volume $V$ is given by

$$
P(0, \rho)=e^{-\rho V} \text {. }
$$

If we now fix a desired confidence level $\gamma$ (in our case $\gamma=99.5 \%$ ) and define the value $\rho_{u}^{N}$ implicitly via

$$
\sum_{m=0}^{N} P\left(m, \rho_{u}^{N}\right)=1-\gamma
$$

then it follows from (1) that for any value of the impurity density $\rho$ bigger than $\rho_{u}^{N}$, the probability of finding $\mathrm{N}$ or fewer inclusions within a volume $V$ is below $1-\gamma=0.5 \%$. In the special case that no inclusions were found equation (2) can be solved explicitly and yields

$$
\rho_{u}^{0}=\frac{-\ln (1-\gamma)}{V} \approx \frac{5.3}{V},
$$

If a performed scan of the volume $V$ yields $\mathrm{N}$ or fewer inclusions, we can therefore conclude that

$$
\rho<\rho_{u}^{N}
$$

with a confidence of $\gamma=99.5 \%$. Subject to this confidence level, the $\rho_{u}^{N}$ defined in equation (3) establishes an upper bound for the unknown inclusion density $\rho$.

The grain size distribution function. So far we have assumed that there exists only one type of inclusion. In reality, inclusions can vary in grain size and composition. For simplicity, let us first study the case where the chemical composition of the inclusion is fixed (for example, $\mathrm{Fe}_{3} \mathrm{O}_{4}$ as worst case scenario in our case) and all inclusions are spherical. However, we do not make any prior assumptions with regards to the inclusion grain size. We can thus introduce a grain size

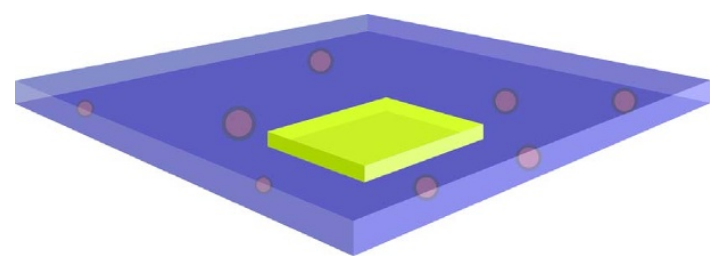

Figure 1 Schematic illustration of a scan of a volume $V$ (small yellow box). What conclusions can we draw, if no inclusions (red balls) are detected during the scan? 
distribution function $p_{G}(d)$, such that for small $\Delta d$ the quantity $p_{G}(d) \Delta d$ is the density of inclusions with diameters in the range $[d$, $d+\Delta d$ ]. We can now generalize the upper bound (3) for the case of a size dependent distribution function as follows: assume that a scan of a certain volume $V$ established that there are $\mathrm{N}$ inclusions present which are larger than a minimal detectable size $d_{m}$. Then we can conclude that

$$
\int_{d_{m}}^{\infty} d q p_{G}(q)<\rho_{u}^{N}
$$

with confidence level $\gamma=99.5 \%$.

Series of measurements. So far we have considered only one volume scan. In practice, any analysis will employ a number $(K)$ of scans with different volume sizes $V_{k}$ possessing different minimal detectable grain sizes $d_{k}$ where the index $k=1, \ldots, K$ enumerates the individual scans starting at large $d_{k}$ (i.e. $d_{k}>d_{k+1}$ ) that yielded $N_{k}$ or fewer inclusions. Furthermore we assume that there is a physical upper bound $d_{0}>d_{1}$ on the grain size. For a given grain size distribution function $p_{G}(d)$ we can define the density of inclusions bigger than $d_{k}$ via

$$
\rho_{k}=\int_{d_{k}}^{d_{0}} p_{G}(q) d q
$$

Then the probability of finding $N_{k}$ or fewer inclusions in one particular scan $k$ is given by

$$
P_{k}\left(N_{k}\right)=\sum_{m=0}^{N_{k}} P\left(m, \rho_{k}\right)
$$

The probability of finding fewer than $N_{k}$ inclusions in a sequence of scans from $k=1, \ldots, K$ is given by the product of the individual probabilities

$$
P=P_{1}\left(N_{1}\right) P_{2}\left(N_{2}\right) \cdots P_{K}\left(N_{K}\right) .
$$

Let us now assume that all individual scans establish that no inclusion was detected larger than the respective minimal grain size, $d_{k}$. This is the case for the application at hand and therefore will now be discussed in more detail. The probability for this zero outcome is given by

$$
P(0)=P_{1}(0) P_{2}(0) \cdots P_{K}(0) .
$$

The volume fraction $\varphi_{k}$ of inclusions between sizes $d_{k}$ and $d_{k-1}$ can be calculated from the grain size distribution $p_{G}(d)$ assuming spherical inclusions

$$
\varphi_{k}=\int_{d_{k}}^{d_{k-1}} d q p_{G}(q) \frac{4}{3} \pi\left(\frac{q}{2}\right)^{3} \leq\left[\int_{d_{k}}^{d_{k-1}} d q p_{G}(q)\right] \frac{\pi}{6} d_{k-1}^{3} .
$$

In this step we have employed the worst case scenario that the weight of the grain size distribution function is located at the large inclusion sizes. It therefore follows that there is an upper limit $\varphi_{k, u}$ on the volume fraction $\varphi_{k}$ of inclusions with diameter in the interval $\left[d_{k}\right.$, $d_{k-1}$ ] given by

$$
\varphi_{k}<\varphi_{k, u}=\frac{-d_{k-1}^{3}}{\sum_{j=K}^{k} V_{j}} \frac{\pi}{6} \ln (1-\gamma) \approx 2.8 \frac{d_{k-1}^{3}}{\sum_{j=K}^{k} V_{j}},
$$

which again holds with a confidence level of $\gamma=99.5 \%$.

Here we have focused on spherical inclusions, but the generalization to other shapes is straight forward. For example, the assumption of cylindrical inclusions may be justified if the inclusion diameter exceeds the sample thickness.
Effect of inclusions on remanent magnetisation. In the previous paragraph we established an upper limit of the volume fraction of inclusions in thin films. However, our aim is to establish an upper bound on the contribution of such inclusions on a measurable quantity. For multiferroic materials, the relevant quantity is the remanent magnetisation, which we will discuss now. Given the bound on the volume fraction, we can define the maximal contribution to the remanence from inclusions with diameters in the interval $\left[d_{k}, d_{k-1}\right]$ as

$$
M_{r, k, u}=\varphi_{k, u} M_{r, F}\left(d_{k-1}\right)
$$

Here $M_{r, F}(d)$ is the remanent magnetization of pure $\mathrm{Fe}_{3} \mathrm{O}_{4}$ grains of diameter $d$. For simplicity we assume that $M_{r F}(d)$ vanishes for $<5 \mathrm{~nm}$. Furthermore we set $M_{r, F}(d)=10 \mathrm{emu} / \mathrm{g}$ for $5 \mathrm{~nm}<d<$ $20 \mathrm{~nm}$ and $M_{r, F}(d)=20 \mathrm{emu} / \mathrm{g}$ for $d>20 \mathrm{~nm}$ which are conservative estimates.

Finally we can establish an upper bound for the total contribution from inclusions to the remanent magnetisation as

$$
M_{r, u}=\max _{W_{1}, \cdots, W_{K}}\left\{\sum_{k=1}^{K} W_{k} M_{r, k, u}\right\}
$$

with a confidence level $\gamma$. Here, the parameters $W_{1}, \ldots, W_{\mathrm{K}}$ fulfill the constraint

$$
\sum_{k=1}^{K} W_{k}=1
$$

Therefore we can conclude that the contribution to the magnetization remanence from inclusions $M_{r}$ has an upper bound

$$
M_{r, u}=\max _{k=1 \ldots K}\left\{M_{r, k, u}\right\}
$$

with confidence $\gamma=99.5 \%$.

\section{Microstructural Analysis}

Guided by the statistical analysis detailed above, we designed our analysis measurements taking into account the sizes of the volumes scanned and the minimum size resolution of the EDX. Monte Carlo simulations $s^{21,22}$ of the electron beam matter interactions estimated a cone shaped interaction volume within the $200 \mathrm{~nm}$ film thickness with a base width of less than $200 \mathrm{~nm}$ and the EDX signal coming from a cylinder with roughly $15 \mathrm{~nm}$ diameter (interaction volume $35000 \mathrm{~nm}^{3}$ ). The interaction volume is only dependent on the ebeam energy $(20 \mathrm{kV})$, spot size $(11.5 \mathrm{~nm})$ and material (assuming that the main phase is the main contributor to calculated interactions) which all stay constant across all measurements. Taking all these factors into account a number of EDX maps were taken from the surface varying the surface area between $36 \mu \mathrm{m}^{2}$ and $1,000,000 \mu \mathrm{m}^{2}\left(1 \mathrm{~mm}^{2}\right)$. As the mapping was done at a set resolution of $1024 \times 886$ pixels (max scanning resolution) we can determine the size of one pixel for each surface area. Hence the smallest detectable size of inclusion across all scanned areas is equal to 1 single pixel except for the smallest area where it is within $2 \times 2$ pixels.

A set of 6 samples was made that went through microstructural analysis. All samples are Aurivillius type thin oxide films and their composition is summarised in Tab. 1.

As described above, spinel-phase magnetite $\left(\mathrm{Fe}_{3} \mathrm{O}_{4}\right)^{23,24}$ is the worst case scenario for second phase inclusions in the type of samples we analyzed, but many other oxides exist that could be built from the elements of the main phase, and thus could occur as second phase inclusions. Some of these are also ferromagnetic. Hence it is necessary to scan for concentrations of all the magnetic elements that are present. The difficulty with these elements, however, is that they are also present in the main phase, and the ions in the main phase will 


\begin{tabular}{|lll|}
\hline $\begin{array}{l}\text { Table } 1 \\
\text { analysis }\end{array}$ & Summary of 6 samples that underwent microstructural \\
Sample & \multicolumn{1}{l}{ Nominal composition } & \multicolumn{1}{c}{ Acronym } \\
\hline $\mathbf{1}$ & $\mathrm{TbBi}_{5} \mathrm{Ti}_{3} \mathrm{Fe}_{2} \mathrm{O}_{18}$ & TbBi5TFO \\
$\mathbf{2}$ & $\mathrm{Tb}_{0.5} \mathrm{Bi}_{5.5} \mathrm{Ti}_{3} \mathrm{Fe}_{2} \mathrm{O}_{18}$ & Tb5Bi55TFO \\
$\mathbf{3}$ & $\mathrm{Tb}_{0.05} \mathrm{Bi}_{5.95} \mathrm{Ti}_{3} \mathrm{Fe}_{2} \mathrm{O}_{18}$ & Tb5B595TFO \\
$\mathbf{4}$ & $\mathrm{Bi}_{5} \mathrm{Ti}_{3} \mathrm{Fe}_{0.7} \mathrm{Co}_{0.3} \mathrm{O}_{15}$ & BTF7C3O ${ }^{27}$ \\
$\mathbf{5}$ & $\mathrm{Bi}_{7} \mathrm{Ti}_{3} \mathrm{Fe}_{3} \mathrm{O}_{21}$ & B7TFO \\
$\mathbf{6}$ & $\mathrm{Bi}_{6} \mathrm{Ti}_{2.8} \mathrm{Fe}_{1.52} \mathrm{Mn}_{0.68} \mathrm{O}_{18}$ & B6TFMO \\
\hline
\end{tabular}

produce the majority of the X-ray signal detected for a given element. Figure 2 shows a typical result from a scanned area of $1600 \mu \mathrm{m}^{2}$ in B6TFMO (sample 6). At first, the elemental maps were colour coded (red - $\mathrm{Ti}$, green - Fe or $\mathrm{Mn}$, blue - $\mathrm{Bi}$ ), added up into an RGB image and superimposed onto the SEM image, all done within the Oxford INCA software. Although an average recording of $5 \times 10^{9}$ counts over 72 hours improved the signal-to-noise-ratio by a factor of 71 compared to a standard scan with an average of $1 \times 10^{6}$ total counts, and eliminated all the noise that was inhibiting the visibility of possible inclusions in the maps achieved from shorter scans, the RGB colour coded images did not reveal inclusions instantly. The subtraction of the Bi EDX signal from the EDX signals coming from the $\mathrm{Fe}$ and $\mathrm{Mn}$ allows us to detect subtle variations in the latter from the average value. The resulting maps are shown in figures $2 \mathrm{~d}$ ) Fe $\mathrm{K} \alpha$ map minus $\mathrm{Bi} \mathrm{L} \alpha$ map and e) Mn K $\alpha$ map minus $\mathrm{Bi} \mathrm{L} \alpha$ map respectively which revealed small bright spots with higher Fe and Mn content and larger $(\sim 1 \mu \mathrm{m})$ pale areas with higher Fe content. The subtraction of the spectral imaging data was done under the assumption that the average $\mathrm{Fe}$ and $\mathrm{Mn}$ content in the main phase stays constant over the whole sample and all we are seeking to find are small changes from that average. Very important to note is the stability of the conditions during the $72 \mathrm{~h}$ scan. There is an approx. $100 \mathrm{~nm}$ layer of carbon deposited over $72 \mathrm{~h}$ and this carbon is regularly patterned. Each of the carbon dots represents exactly one pixel in the EDX measurement that was exposed approx. 1100 times.

This method of subtracting the images to eliminate the signal from the Fe in the major phase was applied to all 6 samples. Except for the B6TFMO, all other analyzed samples showed potentially ferromagnetic inclusions of $\mathrm{Fe}_{\mathrm{x}} \mathrm{A}_{\mathrm{y}} \mathrm{O}_{\mathrm{z}}$ (see Figure 3) where $\mathrm{A}$ is substituted by $\mathrm{Tb}$ for the three samples containing $\mathrm{Tb}$ (samples 1-3), by Co for BTF7C3O (sample 4) and without A for B7TFO (sample 5).

We also note that the inclusions are independently distributed. We have tested this by subdividing the images into partitions and count- ing the number of inclusions in each partition. The resulting count histograms, as represented by the typical example in Fig. 3f), show the expected Poisson distribution. It therefore follows that the spatial distribution of the inclusions is random and without detectable correlations, and justifies the basic assumption in the statistical analysis.

The B6TFMO (sample 6) is interesting insofar as small bright spots (100-500 $\mathrm{nm}$ in diameter) with higher Fe and Mn content and larger paler areas $(\sim 1 \mu \mathrm{m}$ in diameter $)$ with higher Fe content have been found (Fig. 2, 4). Notably when comparing the Fe and Mn maps, the 'large pale areas' appear only in the Fe map (see Fig. 4). In order to investigate the microstructure and localization of some of these inclusions across the film thickness, and to correlate this to the magnetic response observed, a site-specific sample preparation was performed using a focused ion beam (FIB) (Fig. S1). At first the locations of a 'small bright spot' (labelled in yellow) and a 'large pale area' (labelled in blue) were marked with a pillar of e-beam deposited carbon (Fig. 5a). After the cross section lamella was successfully lifted out, attached to a copper half-grid and was polished to about $300 \mathrm{~nm}$ thickness the pillars were confirmed to be $250 \mathrm{~nm}$ in diameter and hence the location of the two areas was determined to this accuracy.

With the two types of areas isolated, a STEM-EDX scan was performed as shown in figure $5 b$ ) and c). This measurement confirmed the absence of Mn excess in the 'large pale areas'. TEM imaging, in conjunction with selected area electron diffraction (SAED) and spot EDX measurements confirm this type of inclusion rather to be grains of the main Aurivillius phase with a $40 \%$ increased Fe content which has been associated previously to be the most probable cause of the magnetic behaviour of the sample ${ }^{25}$.

TEM imaging, in conjunction with SAED and spot EDX ${ }^{25}$ measurements confirm the 'small bright spots' as mangano-wüstite $\left(\mathrm{Mn}_{0.56} \mathrm{Fe}_{0.44} \mathrm{O}\right)$. This is reported to be non-ferromagnetic, antiferromagnetic below $150 \mathrm{~K}^{26}$ and hence not to be responsible for the magnetic behaviour of the sample. In conclusion, the inclusions found in the B6TFMO are not ferromagnetic. Hence their impact on the main phase magnetism is zero supported by statistical analysis which is elaborated in the Discussion.

The link to the smallest volume in the statistical analysis for the B6TFMO sample consists of additional (168) bright field TEM images that were taken along a $30 \mu \mathrm{m}$ long lamella from a randomly chosen location for the structural analysis. There have been no inclusions observed in a volume of $0.32 \mu \mathrm{m}^{3}$. Every image shows smooth single crystalline Aurivillius phase material with grains touching each other smoothly and tightly (Fig S2). In our former studies of similar samples $\left(\mathrm{BTF} \mathrm{C} 3 \mathrm{O}^{27}\right.$ ) following such a "random" sampling approach, we were able to identify and image in detail the micro-

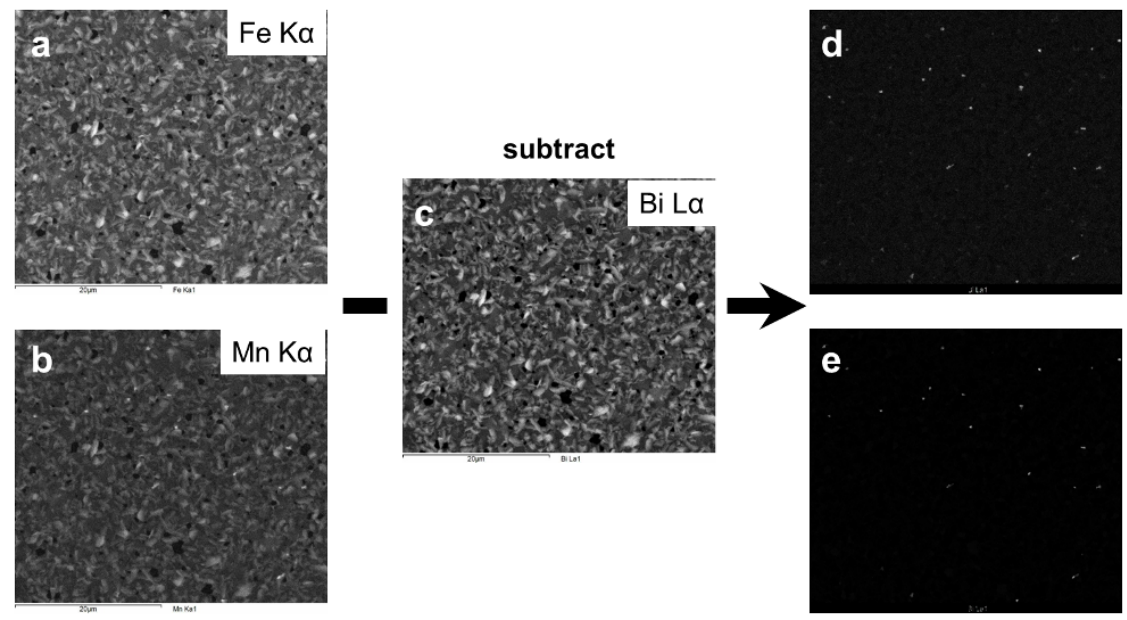

Figure $2 \mid$ Example of EDX signal subtraction in case of B6TFMO sample: (a) Fe K $\alpha$ intensity map, (b) Mn K $\alpha$ intensity map, (c) Bi L $\alpha$ intensity map. The results of subtraction of main phase bound $\mathrm{Fe}$ and $\mathrm{Mn}$ are shown in (d) and (e) respectively. 


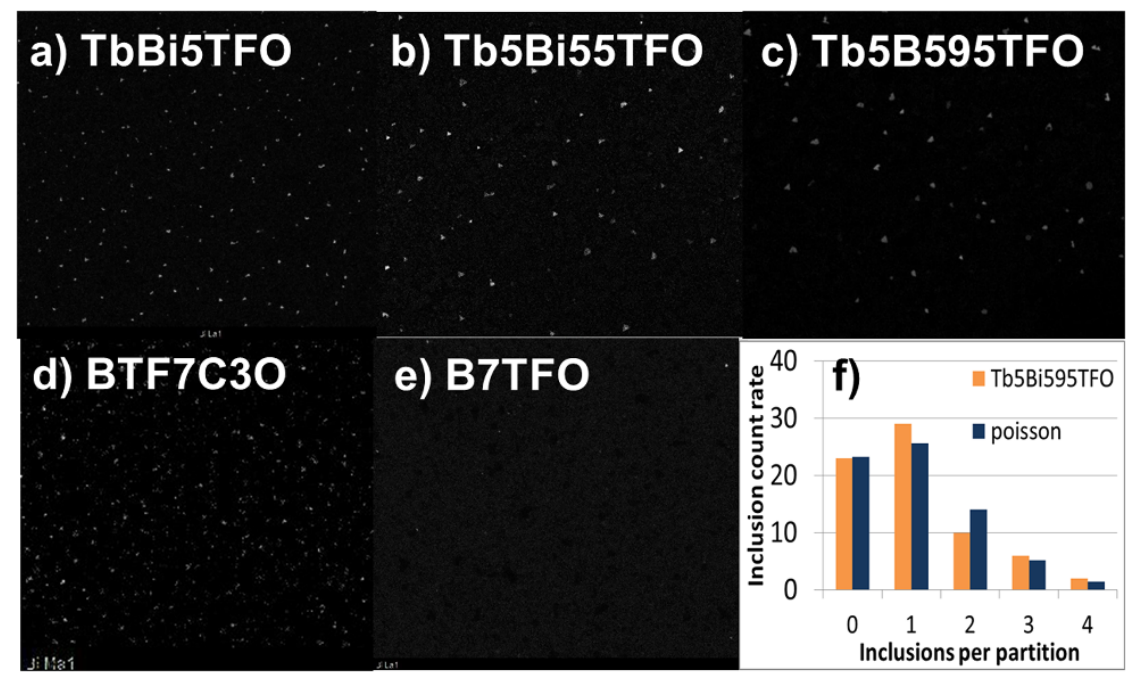

Figure $3 \mid$ (a)-(e) EDX surface maps for samples 1-5 respectively. Main phase bound Fe subtracted as per Fig. 2 . The small bright spots visible are potentially ferromagnetic $\mathrm{Fe}_{\mathrm{x}} \mathrm{A}_{\mathrm{y}} \mathrm{O}_{\mathrm{z}}$ inclusions. (f) exemplary inclusion-count-rate histogram of (c) subdivided into 90 partitions, where the $\mathrm{x}$-axis is the number of counts in a partition and the $y$-axis represents the number of partitions containing that number of counts. The theoretical poisson distribution is depicted for comparison.

structure and composition of inclusions, similar to the type of second phase material being sought here. Hence for the sample under study in this report we can confirm that there are none. The resolution at the chosen magnification in the TEM was such that inclusions down to $2 \mathrm{~nm}$ would be identified, if present.

\section{Discussion}

The main question asked regarding the multiferroic oxide thin films is: 'Are there any ferromagnetic inclusions?'. This is the single most critical factor in the analysis of new single phase multiferroic materials because the magnetic response is particularly sensitive to the amount of such inclusions. To answer this question we developed a comprehensive framework based on linking the experimental EDX data from SEM and TEM scans with the numerical statistical analysis described above. The analytical framework we developed is schematically described in a flow chart (figure 6). The first step is in finding the optimum experimental analytical technique that can obtain spatially resolved maps of elemental compositions. EDX analysis is an obvious choice as it has the spatial resolution necessary to localize the impurities, and reasonably-good detection limits to most elements ( $Z$ $\geq 10$ ). Moreover, EDX analysis on SEM in the case of flat substrates does not require any extensive and elaborate sample preparation.
The data acquisition (productivity of detection) has increasingly being improved by the introduction of large area solid phase detectors (SSD), increasing the solid angle of detection and automation procedures. In the next step, the obtained results of the experimental analysis can be treated as: YES/NO in regard to second phase detected. Once the choice is made the statistics need to be developed in order to supply the parameters that provide the input for the design of the experiment. In the section Statistical Analysis we demonstrate in detail the major steps in the development of the statistical analysis; starting at analyses of the statistical significance of a single measurement of a given sample volume and at a fixed resolution, we then proceeded to evaluate a series of measurements at varying volumes and resolutions. This methodology finally gives the possibility to conclude, in this case with a statistical confidence level of $99.5 \%$, that a physical effect (e.g. the magnetic remanence of the sample) is not caused by undetected inclusions (NO choice feeds into the statistical analysis to calculate the upper limit of impact).

In the case of inclusions being observed (YES outcome), the nature (structural, compositional properties) of these has to be determined in order to answer the next question in the flow chart: 'Do the observed inclusions have an effect on the magnetic remanence of the main phase?' If they do, the percentage of impact needs to be
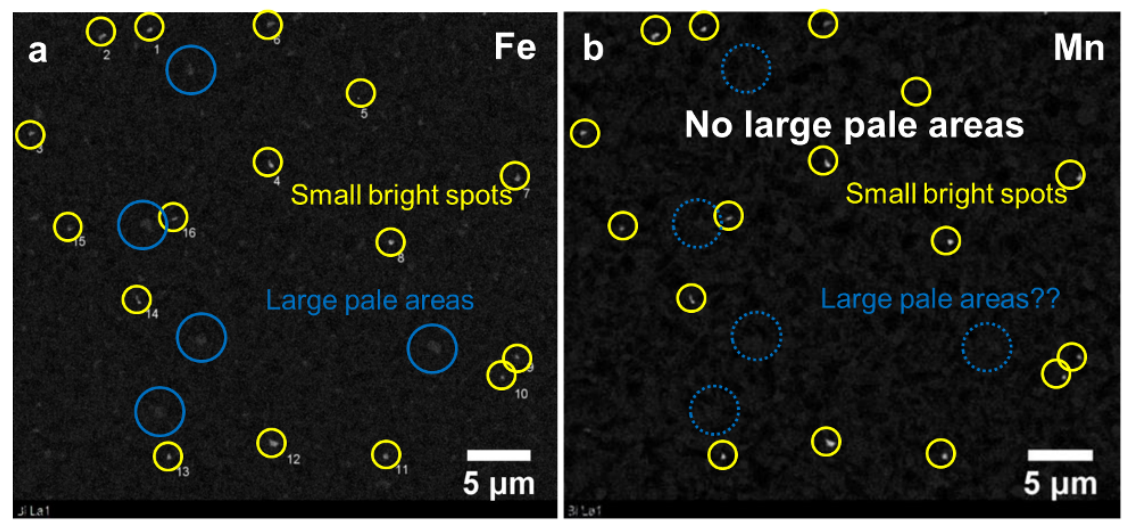

Figure $4 \mid$ A comparison between Fe map (left) and Mn map (right) for the B6TFMO after subtraction of the Bi signal reveals the absence of the 'large pale areas' in the Mn map. 


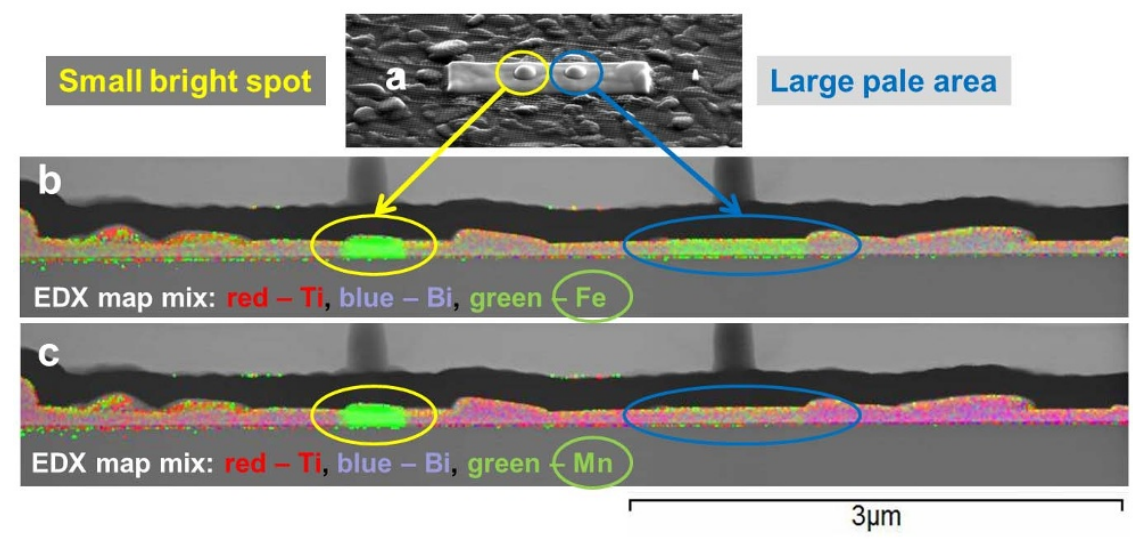

Figure 5 Elemental mapping of a cross section of B6TFMO prepared from the area shown in (a). (b) Fe is coded is green colour; (c) Mn is coded in green colour.

calculated. For example, the presence of second phase magnetic inclusions at a volume fraction of $3.95 \%$ accounted for all of the observed magnetization in the BTF7C3O thin films reported previously ${ }^{27}$. Though any trace of ferromagnetic inclusions within asserted multiferroic materials might raise concerns, the multiferroic property of a new material can still be established with a given confidence level, if the calculated impact of the inclusions seen is minor.

In the case of no inclusions being observed ( $\mathrm{NO}$ case) the contribution of inclusions to the remanence can be estimated statistically. As a worked example we have performed on the B6TFMO sample a number of measurements (labelled $k=1, \ldots, 8)$ at varying detection limits $\left(d_{k}=1 \mu \mathrm{m}, \ldots 2 \mathrm{~nm}\right)$ and scanning volumes $\left(V_{k}=\right.$ $\left.200000 \mu \mathrm{m}^{3}, \ldots, 0.32 \mu \mathrm{m}^{3}\right)$ (see Tab. 2). It is not possible to scan the whole sample at the smallest resolution, and therefore the possibility for ferromagnetic inclusions cannot be excluded with certainty.

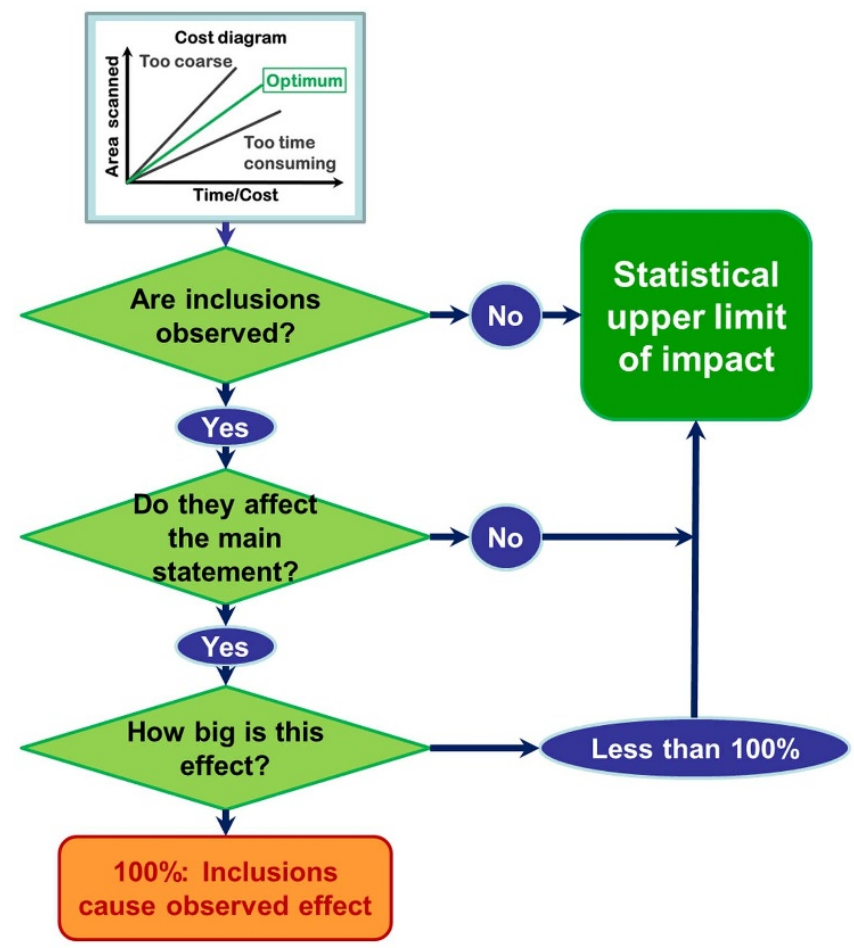

Figure $6 \mid$ The whole process flow of the new generic methodology for localization, identification and grain size distribution of inclusions in thin films with high confidence level.
However, we can use the approach described in section Statistical Analysis to provide an upper bound for the contribution of undetected inclusions to the remanence of the sample with a confidence level of $99.5 \%$. The worst case scenario in the worked example of B6TFMO would be undetected $\mathrm{Fe}_{3} \mathrm{O}_{4}$ inclusions and we therefore calculate the upper bound for this case. We assume that the remanent magnetization is directly proportional to the volume fraction of the inclusions. A direct correlation between the volume fraction of magnetic impurity inclusions and the observed magnetisation was previously demonstrated for the BTF7C3O sample ${ }^{27}$. We also assume that the magnetic inclusions do not interact with the Aurivillius phase matrix. This was justified by the lack of exchange-bias interactions at $2 \mathrm{~K}$ $( \pm 5 \mathrm{~T}$ ) between the 4-layered BTF7C3O sample (Aurivillius phase is

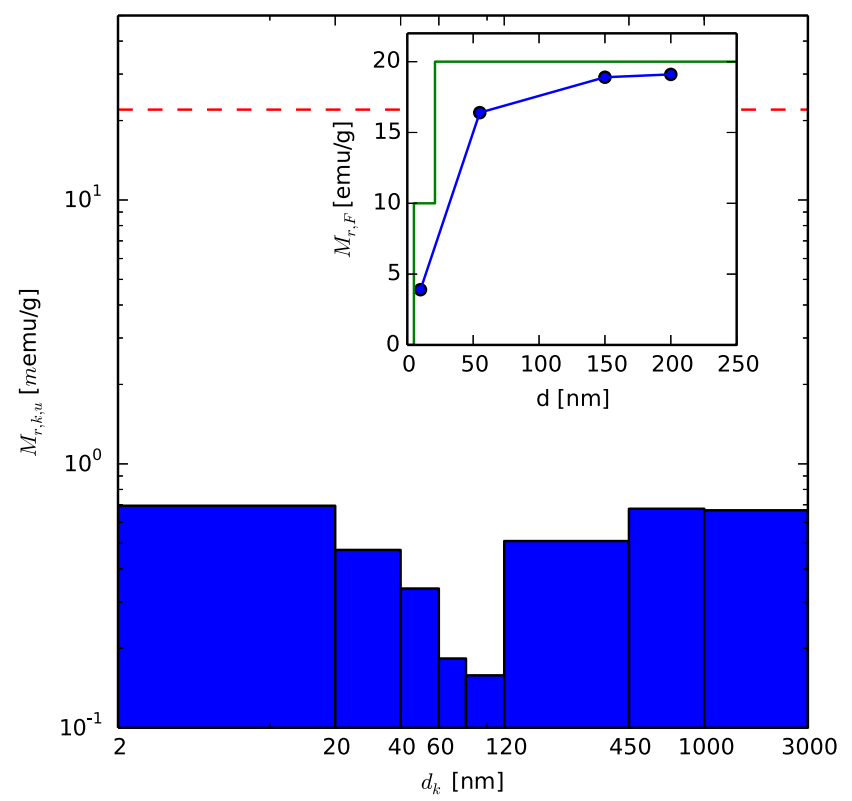

Figure $7 \mid$ The blue bars indicate the maximal contribution $M_{r, k, u}$ to the remanent magnetization from possible unobserved inclusions with diameters in the interval $\left[d_{k}, d_{k-1}\right]$ as obtained from equation (5). The measured remanence of $22 \mathrm{memu} / \mathrm{g}$ for the B6TFMO sample is shown by the dashed red line. The inset shows the remanent magnetization $M_{r, F}$ of $\mathrm{Fe}_{3} \mathrm{O}_{4}$ as function of grain size. Here the blue line shows literature data from reference 23 and reference 24 . Because there are only a few literature values, we use a conveniently chosen (piecewise constant) green line which conservatively overestimates the remanent magnetization for all grain sizes d. 
Table 2 | Performed Volume Scans for sample B6TFMO

\begin{tabular}{lccccc} 
Method & $K$ & $V_{\text {olume }}(\mu \mathrm{m} 3)$ & smallest diameter $d_{k}(\mathrm{~nm})$ & $\Phi_{k}(\%)$ & $M_{r, k, u}(\mathrm{meme} / \mathrm{g})$ \\
\hline Surface EDX & 1 & 200000 & $1000\left(d_{0}=3 \mu \mathrm{m}\right)$ & 0.003 & 0.67 \\
Surface EDX & 2 & 18000 & 450 & 0.003 & 0.68 \\
Surface EDX & 3 & 6000 & 120 & 0.003 & 0.001 \\
Surface EDX & 4 & 450 & 80 & 0.001 & 0.16 \\
Surface EDX & 5 & 120 & 60 & 0.002 & 0.18 \\
Surface EDX & 6 & 28 & 40 & 0.002 & 0.34 \\
Surface EDX & 7 & 7.2 & 20 & 0.007 & 0.47 \\
TEM & 8 & 0.32 & 2 & 0.69 \\
\hline
\end{tabular}

antiferromagnetic at $2 \mathrm{~K}$ ) and the ferrimagnetic $\mathrm{CoFe}_{2-\mathrm{x}} \mathrm{Ti}_{\mathrm{x}} \mathrm{O}_{4}$ impurity inclusions (present at 3.95 vol.\%) ${ }^{28}$. The effects of longrange magnetic interactions between unseen magnetic particles $(<2 \mathrm{~nm})$ were also excluded, since there was no evidence of a super-paramagnetic blocking temperature for B6TFMO ${ }^{25}$.

To simplify the calculation we assume that the remanent magnetization of $\mathrm{Fe}_{3} \mathrm{O}_{4}$ inclusions as a function of the grain size $\left(M_{r, k, u}\right)$ is given by the green line in the inset of Fig. 7, which is chosen to be consistently above the literature values. We then calculate the values $M_{r, k, u}$ which are the upper limits for the remanence contribution for each resolution interval $\left[d_{k}, d_{k-1}\right]$ according to formula (5). The result is shown in the main panel of Fig. 7. We observe that all values $M_{r, k, u}$ are well below the experimentally measured remanent magnetisation of our sample (red dashed line of Fig. 7). For the upper limit of the remanence by unobserved $\mathrm{Fe}_{3} \mathrm{O}_{4}$ inclusions we therefore find a value of $M_{r, u}=0.69 \mathrm{memu} / \mathrm{g}$ with a confidence level $99.5 \%$. This $M_{r, u}$ is only $3 \%$ of the measured magnetic remanence. This compares with a value of $13 \%$ as reported $\mathrm{in}^{25}$, for which the $M_{r, u}$ was calculated using a more restricted dataset as noted above. Following section Statistical Analysis this implies that the measured remanence is not due to undetected $\mathrm{Fe}_{3} \mathrm{O}_{4}$ inclusions. This bound does not critically depend on the inclusions being spherical, for the errors made in the case of cubic, cylindric or ellipsoidal are minimal as the method of detection works with volume fractions independent of the shape of the inclusions.

In conclusion we can say that we have developed a comprehensive and reliable method to detect, localize, isolate and analyse $\mathrm{nm}$ - and $\mu \mathrm{m}$ inclusions in multiferroic thin films and calculated their impact on the properties of the main phase. More importantly this newly developed analytical methodology fills the gap between $\mu \mathrm{m}$ inclusions and atomic scale inclusions. We are confident that this method can be applied to materials where a functional property (electrical, optical etc.), might be confounded by a second phase with dimensons in the $\mathrm{nm}$ and $\mu \mathrm{m}$ range provided that the statistical design of the measurement is adapted to the particular problem and material system in question.

\section{Methods}

Thin film samples of Aurivillius type have been grown on $c$-plane sapphire substrates by chemical solution deposition method ${ }^{25,29}$ in order to investigate its potential as a single phase multiferroic material.

Surface EDX mappings and cross section sample preparation required a thin $\mathrm{Au}$ coating $(<20 \mathrm{~nm})$ to reduce the high surface charging of the films. Cross sections were prepared using a DualBeam focused ion beam (FIB) FEI Helios NanoLab $600 \mathrm{i}^{30}$. After the Au sputter coating a $60 \mathrm{~nm}$ layer of amorphous carbon and $300 \mathrm{~nm}$ Pt layer were deposited within the DualBeam FIB by electron beam induced deposition and $2 \mu \mathrm{m}$ thick Pt layer with ion beam induced deposition. These three layers have been grown for protection before the milling process. A $30 \mu \mathrm{m}$ long lamella was prepared and thinned down to $40-60 \mathrm{~nm}$ for the TEM analysis. The thinning at $30 \mathrm{kV}$ was finished by polishing at $5 \mathrm{kV}$ and $2 \mathrm{kV}$ to reduce the ion-beam induced damage to a less than $2 \mathrm{~nm}$ thin layer on both sides ${ }^{31}$.

TEM analysis was done in a JEOL JEM-2100 operating at $200 \mathrm{kV}$ in bright field condition for imaging and selected area electron diffraction (SAED) mode to record diffraction pattern of single grains. Crystal Studio was used to determine the crystal structure by comparing the simulated electron diffraction pattern with the recorded.
All EDX measurements were recorded in the FEI Helios NanoLab 600i operating at $20 \mathrm{kV}$ and $1.4 \mathrm{nA}$ with an attached Oxford X-Max 80 detector. The spectra were analysed with the Inca software.

1. Jercinovic, M., Williams, M., Allaz, J. \& Donovan, J. in IOPConfSer:MaterSciEng. 012012 (IOP Publishing, 2012).

2. Watanabe, M. \& Williams, D. Atomic-level detection by X-ray microanalysis in the analytical electron microscope. Ultmi 78, 89-101 (1999).

3. Carlson, T. A. Photoelectron and auger spectroscopy. PhT 29, 53, doi:10.1063/ 1.3023614 (1975).

4. Benninghoven, A., Rudenauer, F. \& Werner, H. W. Secondary ion mass spectrometry: basic concepts, instrumental aspects, applications and trends. (Wiley, 1987).

5. Khuri-Yakub, B. Scanning acoustic microscopy. Ultra 31, 361-372 (1993).

6. Wong, Y., Thomas, R. \& Pouch, J. Subsurface structures of solids by scanning photoacoustic microscopy. ApPhL 35, 368 (1979).

7. Goldstein, J. et al. Scanning electron microscopy and X-ray microanalysis. (Springer, 2003).

8. Kelly, T. F. \& Miller, M. K. Atom probe tomography. RScI 78, 031101 (2007)

9. Williams, D. B., Papworth, A. J. \& Watanabe, M. High resolution X-ray mapping in the STEM. JElectronMicrosc 51, S113-S126 (2002).

10. Servanton, G., Pantel, R., Juhel, M. \& Bertin, F. Two-dimensional quantitative mapping of arsenic in nanometer-scale silicon devices using STEM EELS-EDX spectroscopy. Micron 40, 543-551 (2009)

11. Muller, D. et al. The electronic structure at the atomic scale of ultrathin gate oxides. Natur 399, 758-761 (1999).

12. Vaz, C. A., Hoffman, J., Ahn, C. H. \& Ramesh, R. Magnetoelectric coupling effects in multiferroic complex oxide composite structures. AdvMater 22, 2900-2918 (2010).

13. Scott, J. Data storage: Multiferroic memories. NatureMater 6, 256-257 (2007).

14. Ramesh, R. Magnetoelectrics: Making metallic memories. NatureNanotech 5, 761-762 (2010)

15. Wang, L. et al. Electric control of magnetism at room temperature. SciRep 2 (2012).

16. Bibes, M. Nanoferronics is a winning combination. NatureMater 11, 354-357 (2012).

17. Rao, C., Sundaresan, A. \& Saha, R. Multiferroic and Magnetoelectric Oxides: The Emerging Scenario. JPhysChemLett 3, 2237-2246 (2012).

18. Iwai, H. Roadmap for $22 \mathrm{~nm}$ and beyond. MiEng 86, 1520-1528 (2009).

19. Genç, A., Cheng, H., Winterstein, J., Pullan, L. \& Freitag, B. 3D Chemical Mapping using Tomography with an Enhanced XEDS System. MicroscAnal 24, 23-25 (2012).

20. Longo, P. \& Twesten, R. D. Fast STEM Spectrum Imaging Using Simultaneous EELS and EDS. Microscopy Today 21, 28-33 (2013).

21. Drouin, D. et al. CASINO V2.42-A Fast and Easy-to-use Modeling Tool for Scanning Electron Microscopy and Microanalysis Users. Scanning 29, 92-101, doi:10.1002/sca.20000 (2007).

22. Hovington, P., Drouin, D. \& Gauvin, R. CASINO: A new Monte Carlo code in C language for electron beam interaction-part I: Description of the program. Scanning 19, 1-14 (1997).

23. Guan, N., Wang, Y., Sun, D. \& Xu, J. A simple one-pot synthesis of singlecrystalline magnetite hollow spheres from a single iron precursor. Nanot 20, 105603 (2009).

24. Goya, G., Berquo, T., Fonseca, F. \& Morales, M. Static and dynamic magnetic properties of spherical magnetite nanoparticles. JAP 94, 3520-3528 (2003).

25. Keeney, L. et al. Magnetic Field-Induced Ferroelectric Switching in Multiferroic Aurivillius Phase Thin Films at Room Temperature. Journal of the American Ceramic Society 96, 2339-2357, doi:10.1111/jace.12467 (2013).

26. Hope, D. A., Cheetham, A. K. \& Long, G. J. A neutron diffraction, magnetic susceptibility, and Moessbauer-effect study of the (manganese iron) oxide ((MnxFe1-x) yO) solid solutions. InCh 21, 2804-2809 (1982). 
27. Keeney, L. et al. Room temperature ferroelectric and magnetic investigations and detailed phase analysis of Aurivillius phase $\mathrm{Bi}_{5} \mathrm{Ti}_{3} \mathrm{Fe}_{0.7} \mathrm{Co}_{0.3} \mathrm{O}_{15}$ thin films. JAP 112, 052010 (2012).

28. Keeney, L., Maity, T., Roy, S. \& Whatmore, R. W. unpublished work.

29. Faraz, A. \& Keeney, L. unpublished.

30. Giannuzzi, L. \& Stevie, F. A review of focused ion beam milling techniques for TEM specimen preparation. Micron 30, 197-204 (1999).

31. Giannuzzi, L. A., Geurts, R. \& Ringnalda, J. 2 keV Ga + FIB milling for reducing amorphous damage in silicon. MiMic 11, 828-829 (2005).

\section{Acknowledgments}

The authors would like to acknowledge financial support by Science Foundation Ireland (SFI) under the FORME Strategic Research Cluster Award number 07/SRC/I1172, Starting Investigator Research Grant (09/SIRG/I1621) and SFI 09/SIRG/I1615.

\section{Author contributions}

L.K. synthesized the sample, MS undertook the experimental analysis with the advice of N.P. and J.D.H. A.A. devised and conducted the statistical analysis. R.W.W. conceived the study of the base material and the use of the statistical analysis to place confidence limits on the results, and supervised the project. M.E.P. managed the parent project FORME and contributed to the discussions around the base program. The manuscript was written with contributions from M.S., A.A., L.K., N.P. and R.W.W. and all authors have read and given approval to the final version of the manuscript.

\section{Additional information}

Supplementary information accompanies this paper at http://www.nature.com/ scientificreports

Competing financial interests: The authors declare no competing financial interests.

How to cite this article: Schmidt, M. et al. Absence of Evidence $\neq$ Evidence of Absence: Statistical Analysis of Inclusions in Multiferroic Thin Films. Sci. Rep. 4, 5712; DOI:10.1038/ srep05712 (2014)

(2) This work is licensed under a Creative Commons Attribution-NonCommercialNoDerivs 4.0 International License. The images or other third party material in this article are included in the article's Creative Commons license, unless indicated otherwise in the credit line; if the material is not included under the Creative Commons license, users will need to obtain permission from the license holder in order to reproduce the material. To view a copy of this license, visit http:// creativecommons.org/licenses/by-nc-nd/4.0/ 